\title{
Cartographie de sols et de paysages en Suisse occidentale
}

L'Auteur, professeur de géographie physique et de pédologie à l'Université d'Amsterdam, tient à exprimer sa reconnaissance sincère à M. J. Eschmann et au Prof. JL. Piveteau, de Fribourg, pour la part qu'ils ont prise au travail de rédaction de cet article.

\section{Sols, terres et paysages}

On constate partout un grand intérêt pour l'étude des sols, caractérisés comme un phénomène naturel spécifique ou comme un facteur écologique dominant. En tant que phénomène naturel, ils constituent «un complexe dynamique tridimensionnel occupant la partie superficielle de l'écorce terrestre et supportant des végétaux. Etant le résultat d'interactions de durée variable entre le climat, les organismes vivants (y compris l'activité humaine), les matériaux-mères et le relief (y compris l'hydrographie), ils présentent des propriétés différentes de celles des matériaux rocheux sous-jacents. Les sols se distinguent entre eux par leurs caractères internes, leur gradient, leur complexité de pente, leur microtopographie, leur teneur en pierres ou les caractéristiques rocheuses de leur surface» (d'après BRINKMANN and SMYTH, 1973). Un sol présente donc un profil et une surface; il constitue un morceau de paysage d'une certaine profondeur, cette dernière étant déterminée par les processus de pédogénèse et le degré d'enracinement des plantes. Il est également un écosystème complexe incluant des communautés faunistiques (par exemple les vers de terre) et floristiques (par exemple les bactéries). Il est donc évident que les sols sont un objet d'étude pour les géographes (qui étudient les paysages), ainsi que pour les biologistes et les agronomes.

Le sol, de même que l'eau, est certainement un facteur écologique de première importance dans tous les paysages. Tous deux sont assez stables pour exercer un effet durable sur les milieux de tous les organismes terrestres. Par ailleurs, ils présentent de grandes variations sur de courtes distances, justifiant ainsi le terme de variables-clefs donné par CHORLEY et KENNEDY (1971). On peut même parler d'une "écologie des sols" lorsqu'on étudie les facteurs de la pédogénèse (climat, roche-mère, relief, végétation, action humaine), leurs interactions et l'effet produit sur la formation des paysages et sur les sols.
La géographie physique a un double but. Le premier est d'étudier la genèse des paysages en se fondant sur la description et l'analyse des formes et des matériaux de la surface terrestre. Il s'agit là d'une des branches des sciences naturelles. Le second est d'étudier les paysages en tant que base écologique du milieu humain. La géographie physique est alors une partie essentielle de la géographie tout entière. Le paysage étant la base de tous les écosystèmes, aussi bien naturels que culturels, l'application des recherches dans ce domaine est essentielle pour le développement régional, ainsi que pour l'amélioration et la gestion de l'environnement.

Sans nier l'intérêt scientifique de l'étude des écosystèmes naturels (objet de la biogéographie), nous préférons nous occuper de ceux qui sont en interaction avec la société humaine. La recherche doit alors être entreprise de deux manières: d'un côté l'accent est mis sur les besoins des groupes humains ou des individus, de l'autre il porte sur les ressources offertes par les différentes «terres» appartenant à un paysage. Il s'agit donc d'une recherche scientifique appliquée où apparaît une tension permanente entre ce que la nature peut offrir et ce que l'homme lui demande. Avec, comme intermédiaire, les possibilités d'application de la technologie en vue d'une adaptation tenant compte de la conservation ou du rétablissement de la stabilité écologique indispensable.

Pour mener à bien cette écologie des paysages, il est nécessaire de disposer d'une bonne étude scientifique des paysages considérés comme ressources naturelles, ainsi que d'un système d'évaluation des résultats de ces recherches en vue de leurs limitations, de leurs aptitudes et de leur vocation pour les diverses activités humaines, appelée aussi "classification des terres» (voir VINK, 1963, 1975). En bref, il s'agit d'étudier les terres sous tous les aspects de la définition suivante (d'après FAO, 1972): «un espace de terre» («a tract of land») est défini géographiquement comme une partie spécifique de la surface de la Planète; ses caractères comprennent tous les attributs raisonnablement stables, ou considérés d'une façon prévisible comme cycliques, de la biosphère, verticalement, en dessus et en dessous de cette surface. Il inclut aussi les caractères de l'atmosphère, du sol et des roches sous-

Prof. A.P. A. Vink, Universität Amsterdam, Dapperstraat 115, Amsterdam-Oost (Niederlande) 
jacentes, de l'hydrologie, des populations végétales et animales et des effets des activités anciennes et actuelles, pour autant que ces attributs exercent une influence significative sur les utilisations présentes et futures de l'espace par l'Homme.

Nous n'allons pas contribuer à la discussion fondamentale sur la notion de «paysage». Pour notre propos, un paysage pourrait être défini comme «un arrangement naturel de terres ayant des relations génétiques et écologiques étroites». L'approche génétique mène, entre autres, à la géomorphologie et à la pédogénèse, tandis que la perspective écologique conduit à l'écologie des paysages (Landschaftsökologie, C. TROLL, 1966). Une discussion des divers aspects des recherches en géographie physique a été donnée récemment dans d'autres publications (JUNGERIUS, KOSTER et KWAAD, 1973; vINK, 1974).

\section{Levers de terrain et recherches en laboratoire}

Nos recherches en Suisse occidentale ont pour but principal la formation pratique et théorique de nos étudiants de 2 ème cycle en géographie physique. Elles sont surtout orientées vers l'écologie des paysages à basse et moyenne altitude, soit jusqu'à $2000 \mathrm{~m}$. En outre, une étude orientée vers la prospection pédologique et la genèse des sols a été faite dans la région de Morat (VAN DER MEER, 1973, 1975). La figure 1 montre les régions étudiées. Les premiers résultats des recherches éco-paysagiques ont paru en 1974 (KLAASEN, 1974; WEVERS, 1974) et 1975 (BLANKERS, 1975; DU PREE, 1975).

Les méthodes utilisées consistent dans l'utilisation de photographies aériennes (VINK, 1963, 1968), dans la prospection systématique de terrains et dans des analyses d'échantillons réalisées avec diverses méthodes. Un grand profit est tiré des études sur la géologie quaternaire effectuées dans ces régions (BURRI, 1962; HANTKE, 1963; PORTMANN, 1955, 1966, 1972). Une amélioration de nos méthodes de recherche a pu être réalisée grâce aux études climatologiques, écoclimatologiques et écopaysagiques de MARR (1970), SCHREIBER (1968) et HAEBERLI $(1968,1971)$. Les études de MEYER DE STADELHOFEN (1967) ont procuré une meilleure connaissance de certains aspects de la géophysique du canton de Vaud. Les investigations archéologiques faites dans le cadre de la 2ème correction des eaux du Jura ont approfondi nos connaissances des développements holocènes (SCHWAB, 1969, SCHWAB et MÜLLER, 1973).

Des recherches du même type sont effectuées en Italie, près de Florence et au sud du Rome (VINK, 1973). Les conclusions générales qu'on peut en tirer ne sont pas contradictoires avec celles qui ont été mises en évidence en Suisse occidentale.

\section{Une prospection pédologique}

Nos prospections pédologiques sont de type «physiographique». Cela signifie que la cartographie ne se limite pas aux profils des sols, mais reproduit également leur situation et les caractères dérivés de celle-ci. La formation des sols se fait selon deux processus: celui de la géogénèse et celui de la pédogénèse. La géogénèse constitue les matériaux-mères, qui proviennent très souvent d'un transport des roches-mères par des agents érosifs et sédimentaires. La pédogénèse est surtout causée par le climat, la végétation et l'eau. Les deux processus se superposent, de sorte que souvent leurs effets ne peuvent être compris que comme un résultat intégré. Ainsi, les différences locales des sols sont toujours fortement influencées par la répartition des différents matériaux-mères, caractérisés par leurs formes de relief et leurs propriétés de drainage, qui constituent les conditions locales de la pédogénèse. L'évolution d'un sol particulier résulte des processus propres à un complexe géo-pédogénétique, qui détermine la succession des écosystèmes naturels et peut même avoir une forte influence sur l'écologie des systèmes culturels. La confection de cartes pédologiques physiographiques est alors la méthode qui convient le mieux pour les buts fixés.

La carte de la région de Morat (VAN DER MEER, 1973, 1974) est un exemple d'une telle cartographie. L'extrait reproduit ici (figure 2 et sa légende au tableau 1) est à l'échelle originale de 1:50000. D'une manière générale, on trouve peu de différenciations entre les grands groupes de sols: Fluvisols, Arenosols, Cambisols, Gleysols, Luvisols, Histosols. Seul le paysage "Alluvial» fait exception. Les autres paysages fournissent cependant des distinctions d'une certaine importance, surtout du point de vue "édaphologique». On pourrait évidemment distinguer moins d'unités, ce qui rendrait la carte plus visible. Mais on perdrait alors 
en information et il ne serait plus possible, sans travailler à une échelle beaucoup plus grande $(1: 5000)$, de distinguer les différentes «unités pédogénétiques pures».

Grâce à une combinaison de données sur la pédogénèse, la géogénèse et le relief, la carte permet de tirer certaines conclusions quant à la genèse des sols. Ainsi, les Luvisols les mieux développés se rencontrent dans trois types de paysages: Haute Molasse, Vallées et Alluvial. Leur durée de formation peut différer considérablement, mais on peut dire que, sous ce climat, elle s'est étalée sur quelques milliers d'années. Les

Tableau 1: Extrait de la légende de la carte pédologique de la région de Morat (VAN DER MEER, 1973), valable pour la figure 2.

\begin{tabular}{|c|c|c|c|c|c|}
\hline Paysage & $\begin{array}{l}\text { Sym- } \\
\text { bole }\end{array}$ & $\begin{array}{l}\text { Groupe pédologique } \\
\text { (FAO; Dudal, 1970) }\end{array}$ & Texture & $\begin{array}{l}\text { Phase } \\
\text { de pente }\end{array}$ & $\begin{array}{l}\text { Phase } \\
\text { de drainage }\end{array}$ \\
\hline $\begin{array}{l}\text { Haute } \\
\text { Molasse (M) }\end{array}$ & $\begin{array}{l}\text { M1 } \\
\text { à M7 }\end{array}$ & $\begin{array}{l}\text { Luvisols, Gleysols, } \\
\text { Cambisols, Histosols }\end{array}$ & & & \\
\hline $\begin{array}{l}\text { Basse } \\
\text { Molasse (G) }\end{array}$ & $\begin{array}{l}\text { G1 } \\
\text { G2 } \\
\text { G3 } \\
\text { G4 } \\
\text { G5 } \\
\text { G6 }\end{array}$ & $\begin{array}{l}\text { Luvisols + Cambisols } \\
\text { + Gleysols } \\
\text { Luvisols + Cambisols } \\
\text { + Gleysols } \\
\text { Luvisols + Cambisols } \\
\text { + Gleysols } \\
\text { Cambisols + Gleysols } \\
\text { Cambisols + Histosols } \\
\text { Arenosols }\end{array}$ & $\begin{array}{l}\text { limon sableux } \\
\text { peu graveleux } \\
\text { idem } \\
\text { idem } \\
\text { idem } \\
\text { limon sableux } \\
+ \text { tourbe } \\
\text { sable peu } \\
\text { graveleux }\end{array}$ & $\begin{array}{l}\text { assez plat } \\
\text { incliné } \\
\text { à pente } \\
\text { modérée } \\
\text { vallée } \\
\text { assez plat } \\
\text { idem }\end{array}$ & $\begin{array}{l}\text { modéré } \\
\text { bon-pauvre } \\
\text { idem } \\
\text { modéré-pauvre } \\
\text { modéré } \\
\text { (artificiel) } \\
\text { excessif }\end{array}$ \\
\hline $\begin{array}{l}\text { Fluvio- } \\
\text { glaciaire (V) }\end{array}$ & $\begin{array}{l}\text { V1 } \\
\text { à V7 }\end{array}$ & $\begin{array}{l}\text { Luvisols, Cambisols } \\
\text { Gleysols, Histosols, } \\
\text { Arenosols }\end{array}$ & & & \\
\hline Vallées (D) & $\begin{array}{l}\text { D1 } \\
\text { à D8 } \\
\text { D91 } \\
\text { D92 } \\
\text { à D13 }\end{array}$ & $\begin{array}{l}\text { Luvisols, Cambisols, } \\
\text { Gleysols } \\
\text { Cambisols + Gleysols } \\
\text { Cambisols, Gleysols, } \\
\text { Histosols, Regosols, } \\
\text { Lithosols }\end{array}$ & limon sableux & assez plat & modéré \\
\hline Alluvial (A) & $\begin{array}{l}\text { A1 } \\
\text { A2 } \\
\text { A3 } \\
\text { A4 } \\
\text { A5 } \\
\\
\text { A6 } \\
\text { A7 }\end{array}$ & $\begin{array}{l}\text { Luvisols } \\
\text { Arenosols } \\
\text { Cambisols } \\
\text { Gleysols } \\
\text { Gleysols } \\
\\
\text { Histosols } \\
\text { Fluvisols }\end{array}$ & $\begin{array}{l}\text { sable } \\
\text { idem } \\
\text { argile (silteuse) } \\
\text { idem } \\
\text { argile sur } \\
\text { faible tourbe } \\
\text { tourbe } \\
\text { argile }\end{array}$ & $\begin{array}{l}\text { assez plat } \\
\text { idem } \\
\text { idem } \\
\text { idem } \\
\text { dépression } \\
\text { assez plat } \\
\text { idem }\end{array}$ & $\begin{array}{l}\text { excessif } \\
\text { modéré-pauvre } \\
\text { modéré } \\
\text { modéré-pauvre } \\
\text { pauvre } \\
\text { idem } \\
\text { idem }\end{array}$ \\
\hline
\end{tabular}


Fig. 1: Etat des travaux en Suisse occidentale au 1. 9. 1974.

Fig. 2: Extrait de la carte pédologique de la région de Morat (Van der Meer, 1973) Légende: voir tableau 1.

Fig. 3: Extrait de la carte éco-paysagique de la région d'Aigle (Klaasen, 1974) Légende: voir tableau 2.
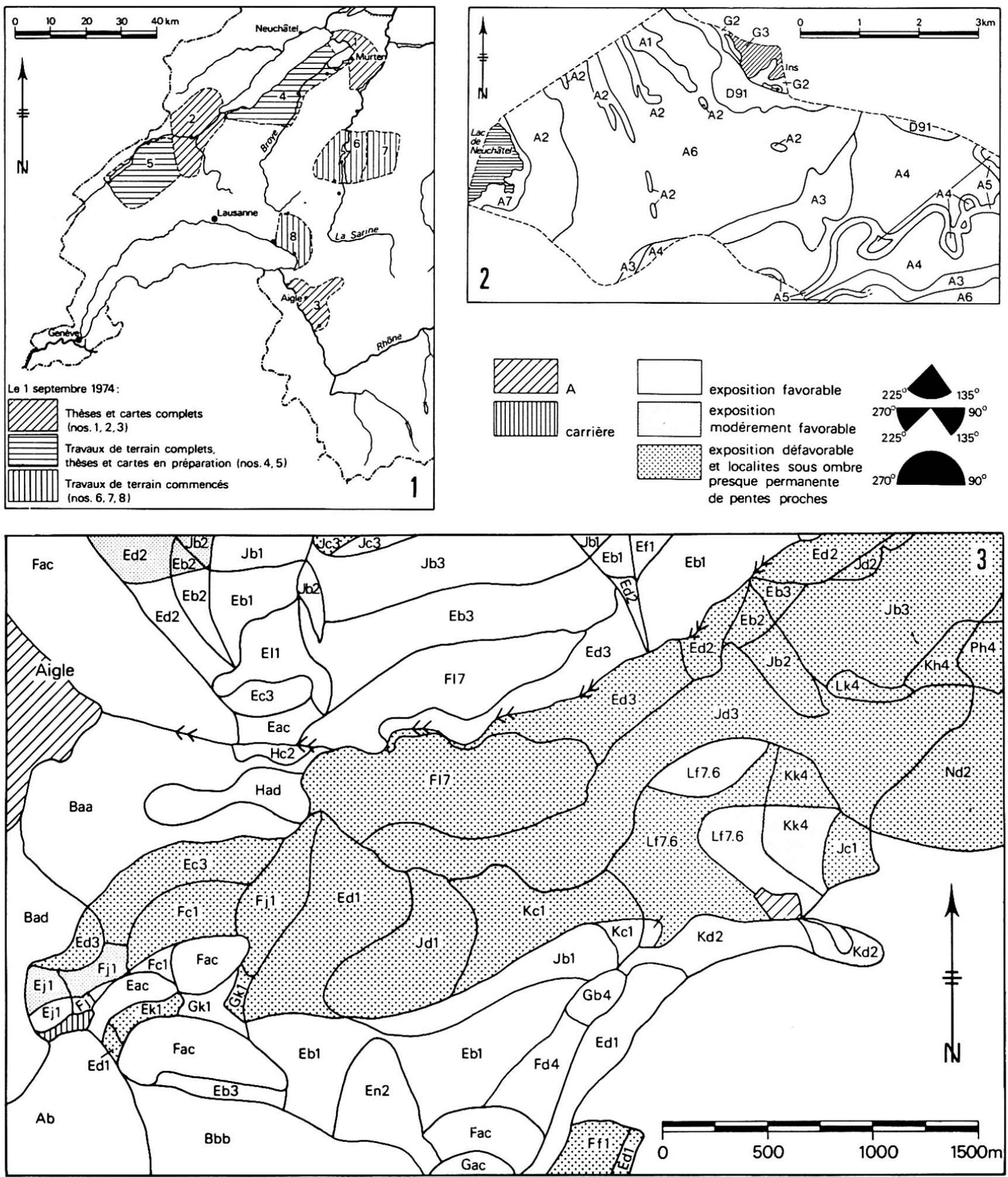
Fig. 4: Evaluation des terres dans la région d'Aigle. Légende: voir tableau 2.
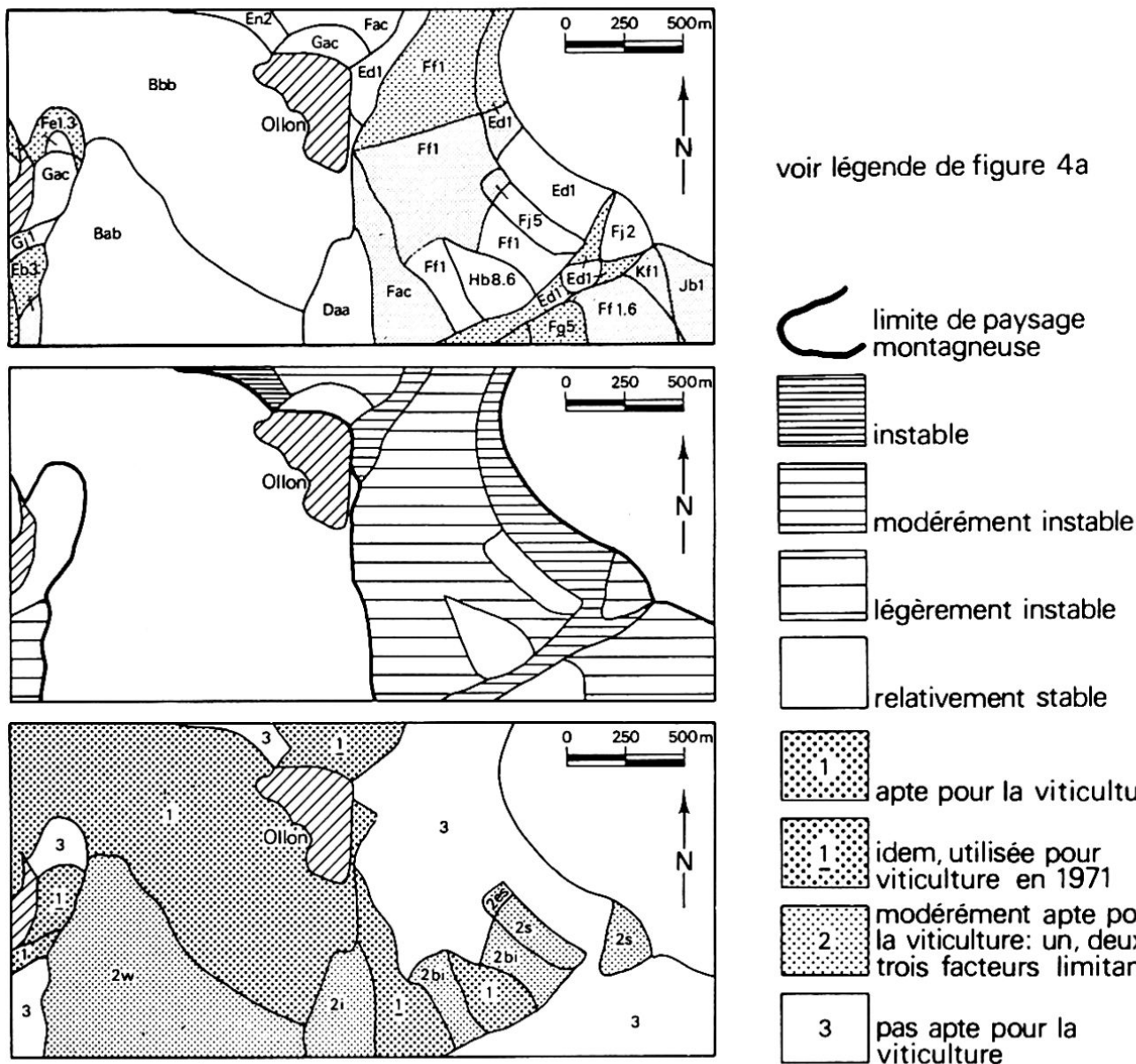

\section{$\because \%$ \\ 6 apte pour la viticulture}

1 idem, utilisée pour $\because 6$ viticulture en 1971

2 la viticulture: un, deux ou

3 pas apte pour la viticulture

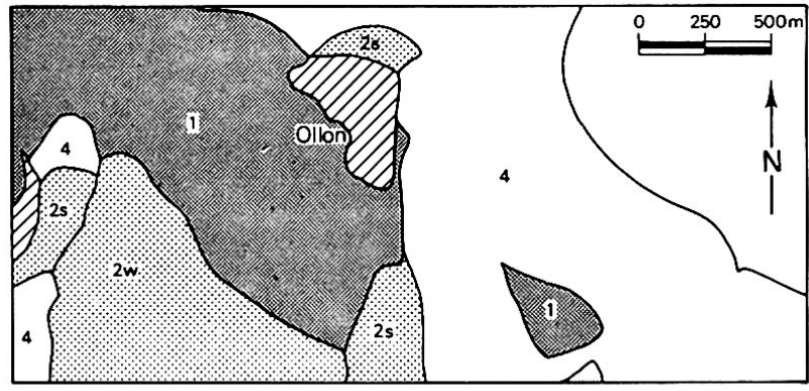

1: camping 2 apte à modérément apte

3 modérément à pas apte pour terrains de camping

$4 \quad$ pas apte pour terrains de camping

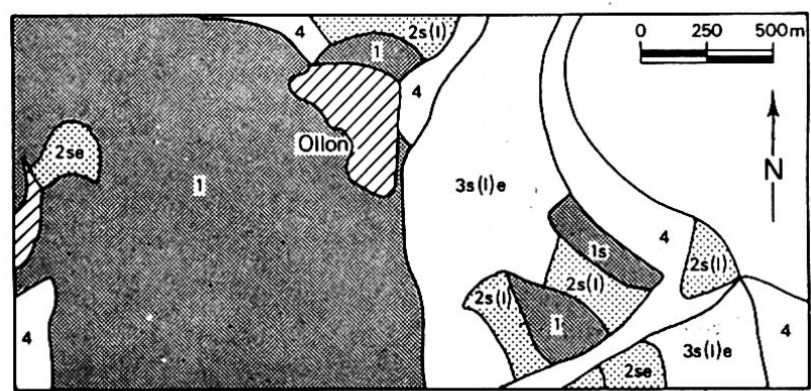

1: apte pour maisons secondaires

2 apte à modérément apte pour maisons secondaires

3 modérément à pas apte pour maisons secondaires

4 pas apte pour maisons secondaires 
mieux développés d'entre eux se trouvent dans la Haute Molasse, soit à plus de $600 \mathrm{~m}$ d'altitude. La pédogénèse a dû $\mathrm{y}$ commencer plus tôt que dans les autres parties de la Molasse. Il devait donc y avoir une végétation plus abondante, ce qui, avec d'autres considérations géomorphologiques, indique un dégel plus précoce du glacier du Rhône würmien. Cette hypothèse est encore renforcée par la présence, dans la Haute Molasse presqu'exclusivement, de sols à "fragipan", horizons spécifiques des processus périglaciaires de pédogénèse (VINK et SEVINK, 1971).

Quant aux Luvisols des terrasses de la Sarine, on ne peut conclure, pour l'instant, qu'à une pédogénèse plus ancienne pour les gradins les plus élevés.

Plus intéressante est la comparaison de la pédogénèse dans les différents cordons litoraux qui bordent le lac de Neuchâtel (A1, A2). Le plus ancien, qui est également le plus élevé et le mieux drainé, est caractérisé par un Orthic Luvisol à très fort lessivage d'argile. Le second groupe de cordons litoraux (A2) passe des Cambisols aux Gleysols en même temps qu'ils deviennent plus récents et moins élevés. Enfin, au bord du lac, on rencontre des Fluvisols qui surmontent des argiles «non-maturées», c'est-à-dire encore gorgées d'eau et plus proches des sédiments originaux que d'un véritable sol. Par une analyse palynologique, et en corrélation avec des données archéologiques, les cordons ont pu être datés. Le plus ancien compte 10000 ans (fin du Pléistocène) et le plus jeune 5000 ans (période "atlantique» de l'Holocène), ce qui correspond assez bien aux données de MÜLLER (SCHWAB et MÜLLER, 1973).

\section{Les prospections écologiques des paysages}

Pour les applications, il faut considérer les levers géomorphologiques et pédologiques dans une optique écologique, donc tenir compte de tous les aspects qui ont une influence sur les milieux des écosystèmes naturels, semi-naturels et culturels. Concernant l'rutilisation des terres» dans son sens le plus large, qui inclut la conservation de la nature et l'utilisation urbaine du sol, on peut même parler d'«écologie de l'utilisation des terres» (VINK, 1975). Dans cette optique, d'autres facteurs écologiques sont à prendre en considération, notamment, dans une région comme la Suisse, la climatologie des paysages, ainsi que l'ont très bien montré SCHREIBER (1968), HAEBERLI $(1969,1971)$ et MARR (1970). En fait, à la base de l'écologie dans tous les paysages suisses, on trouve un complexe composé des facteurs géologique, géomorphologique, hydrologique, climatologique, pédologique, floristique, faunistique et artificiel (canalisations, digues, terrasses, voies de communications, constructions)

Pour garantir les meilleurs résultats, l'analyse et la synthèse de tous ces aspects nécessitent des «levers intégrés» exécutés par une équipe de chercheurs. Il faut y adjoindre les données démographiques, sociologiques et économiques. Enfin, il convient de trouver une base de synthèse grâce à laquelle les différents aspects des «terres» peuvent être cartographiés en vue de l'évaluation (cf. paragraphe suivant) et de la planification. Dans plusieurs pays, cette base figure sur les cartes pédologiques et s'est souvent montrée satisfaisante.

Il existe cependant des régions où les cartes pédologiques ne sont pas les meilleurs indicateurs pour les variables-clefs écologiques. Il s'agit de régions, comme la zone molassique, où les sols présentent peu de variations d'importance écologique, et où les variablesclefs sont plutôt l'altitude, le relief ou l'hydrologie. A cet effet, nous sommes en train de mettre au point un système de cartographie des paysages, appelée «cartographie éco-paysagique» ou "cartographie écologique des paysages», qui est principalement basée sur les idées originales de TROLL (1966). Les travaux sont exécutés en Suisse romande ( $N$ o 2-8 de la figure 1), en Toscane (VINK, 1973) et aux Pays-Bas.

Pour les recherches effectuées en Suisse, de précieuses indications sont tirées des travaux de SCHREIBER (1968) et HAEBERLI, des feuilles de la Carte géologique de la Suisse, ainsi que de données fournies par l'Office d'Urbanisme du Canton de Vaud et par l'Institut de Géographie de Fribourg.

Les études faites dans la région d'Orbe (WEVERs, 1974) et dans celle d'Aigle (KLAASEN, 1974) (cf. figure 3 et sa légende au tableau 2) montrent que les variablesclefs pour le milieu humain sont l'altitude, le relief (tous deux fortement liés au climat local: topoclimat) et les sols. La végétation n'est indiquée que dans ses grandes lignes, d'une part parce qu'elle a fortement été modifiée par l'homme et d'autre part du fait de l'échelle choisie pour la cartographie. Quant à l'hydrologie, elle est surtout mise en relation avec le relief et les sols. Bien que, par manque de données sur l'hydro- 
logie, la végétation et la faune, tous les aspects écologiques n'aient pas pu être indiqués, ces cartes se prêtent à des évaluations pratiques d'une certaine valeur.

\section{Evaluations des sols et des terres}

Les types de cartes décrites dans les deux paragraphes précédents sont indispensables pour la compréhension des processus de formation et d'évolution des paysages. Les applications qui ne seraient pas basées sur de tels documents auraient une nature éphémère. Cependant, les informations qu'elles recèlent ne sont pas facilement utilisables dans la pratique, du fait de leur présentation selon des formes compliquées et une terminologie spécialisée propre aux sciences naturelles. De plus, leur exploitation peut se faire pour des buts très différents, dans une région et à une période données, et sous des conditions socio-économiques et technologiques spécifiques. L'évaluation des terres demande donc une méthodologie particulière. Elle doit traduire les cartes de base en termes compréhensibles pour un nonspécialiste et en extraire les propriétés essentielles pour un but précis. Elle doit enfin indiquer les valeurs d'utilisation relatives, et si possible absolues, des différentes terres, dans les conditions socio-économiques et technologiques d'une région donnée et d'une période définie (VINK, 1963, 1975).

Etant donné les changements de circonstances régionales et mondiales, les cartes d'évaluation ont une validité plus courte que les cartes de base. En principe, on accepte une durée d'une génération (30 ans) pour celles-ci, alors que les cartes d'évaluation ont besoin de révisions, partielles ou totales, tous les cinq à dix ans. La figure 4 montre quelques exemples d'évaluation (ou de classification) des terres dans la région d'Aigle.

Tableau 2: Légende d'une partie de la carte Eco-paysagique de la région d'Aigle (simplifiée d'après KLAASEN, 1974), valable pour la figure 3 .

\begin{tabular}{|c|c|c|c|c|c|c|}
\hline \multicolumn{7}{|c|}{ Paysage: Vallée du Rhône (A/D) } \\
\hline Matériau & $\begin{array}{l}\text { Macrorelief } \\
\text { et pentes }\end{array}$ & $\begin{array}{l}\text { Micro- } \\
\text { relief }\end{array}$ & Sols & Utilisation & $\begin{array}{l}\text { Caractéristiques } \\
\text { additionnelles }\end{array}$ & Symbole \\
\hline $\begin{array}{l}\text { Alluvions } \\
\text { du Rhône } \\
\text { (A) }\end{array}$ & $\begin{array}{l}\text { plat, pentes } \\
<2 \%\end{array}$ & irrégulier & $\begin{array}{l}\text { complexe de Gleysols, } \\
\text { Phaeozems et Cambi- } \\
\text { sols, riches en cal- } \\
\text { caire, texture limo- } \\
\text { neuse à fine }\end{array}$ & $\begin{array}{l}\text { terres } \\
\text { cultivées }\end{array}$ & $\begin{array}{l}\text { modérément } \\
\text { à bien } \\
\text { drainé }\end{array}$ & $\mathrm{Ab}$ \\
\hline \multirow{3}{*}{$\begin{array}{l}\text { Alluvions } \\
\text { des af- } \\
\text { fluents du } \\
\text { Rhône (B) }\end{array}$} & \multirow[t]{2}{*}{$\begin{array}{l}\text { plat, pentes } \\
<2 \%\end{array}$} & plat & $\begin{array}{l}\text { sols profondément } \\
\text { humiques, limoneux, } \\
\text { pierreux }\end{array}$ & viticulture & $\begin{array}{l}\text { très influencé } \\
\text { par la viti- } \\
\text { culture }\end{array}$ & $\mathrm{Baa}$ \\
\hline & & $\begin{array}{l}\text { presque } \\
\text { plat }\end{array}$ & $\begin{array}{l}\text { complexe de Cambi- } \\
\text { sols et Regosols, } \\
\text { sablo-limoneux, } \\
\text { pierreux, riches en } \\
\text { calcaire }\end{array}$ & $\begin{array}{l}\text { terres } \\
\text { cultivées }\end{array}$ & - & $\mathrm{Bad}$ \\
\hline & $\begin{array}{l}\text { pentes à } \\
5 \%(\mathrm{Bd})\end{array}$ & $\begin{array}{l}\text { presque } \\
\text { plat }\end{array}$ & $\begin{array}{l}\text { Cambisols ochriques, } \\
\text { texture limoneuse, } \\
\text { pierreux à } 50 \mathrm{~cm}\end{array}$ & $\begin{array}{l}\text { terres } \\
\text { cultivées }\end{array}$ & - & $\mathrm{Bbb}$ \\
\hline
\end{tabular}




\begin{tabular}{|c|c|c|c|c|c|}
\hline \multicolumn{6}{|c|}{ Paysage: Région des Montagnes (E/S) } \\
\hline \multicolumn{6}{|c|}{ Pentes: très raides $(>30 \%)$ (E. J.N.) } \\
\hline Matériau & $\begin{array}{l}\text { Relief et } \\
\text { pentes }\end{array}$ & Sols & Utilisation & $\begin{array}{l}\text { Erosion/ } \\
\text { stabilité }\end{array}$ & $\begin{array}{l}\text { Sym- } \\
\text { bole }\end{array}$ \\
\hline Moraines & terrasses & de viticulture & viticulture & - & Eac \\
\hline \multirow{9}{*}{$\begin{array}{l}\text { Roches et } \\
\text { matériaux de } \\
\text { pente }\end{array}$} & \multirow[t]{3}{*}{ rectiligne (b) } & \multirow{8}{*}{$\begin{array}{l}\text { Regosol et Lithosol, } \\
\text { pierreux à } 25 \mathrm{~cm}\end{array}$} & \multirow[t]{3}{*}{ forêts } & invisible & $\mathrm{Ebl}, \mathrm{Jbl}$ \\
\hline & & & & érosion visible & $\mathrm{Eb} 2, \mathrm{Jb} 2$ \\
\hline & & & & roches & $\mathrm{Eb} 3, \mathrm{Jb} 3$ \\
\hline & \multirow[t]{2}{*}{ convexe (c) } & & \multirow[t]{2}{*}{ idem } & invisible & $\mathrm{Jc1}$ \\
\hline & & & & roches & $\mathrm{Ec} 3, \mathrm{Jc} 3$ \\
\hline & \multirow[t]{3}{*}{ concave $(d, e)$} & & \multirow[t]{3}{*}{$\operatorname{idem}(d)$} & invisible & Edl, Jdl \\
\hline & & & & visible & $\mathrm{Ed} 2, \mathrm{Nd} 2$ \\
\hline & & & & roches & Ed3 \\
\hline & irrégulier (f) & $\begin{array}{l}\text { Regosol et Lithosol, } \\
\text { pierreux à } 50 \mathrm{~cm}\end{array}$ & idem & invisible & Efl \\
\hline \multirow{3}{*}{$\begin{array}{l}\text { d'origine mo- } \\
\text { rainique }\end{array}$} & convexe (f) & idem & idem & invisible & Ef1 \\
\hline & \multirow[t]{2}{*}{ concave $(\mathrm{k})$} & \multirow[t]{3}{*}{ idem } & idem & \multirow[t]{2}{*}{ idem } & Ek1 \\
\hline & & & prés (1) & & Ell \\
\hline éboulis & $\begin{array}{l}\text { irrégulier, } \\
\text { concave }\end{array}$ & & forêts (n) & visible & En2 \\
\hline \multicolumn{6}{|c|}{ Pentes: raides $(10 \%$ à $30 \%)(F, K, P)$} \\
\hline Matériau & $\begin{array}{l}\text { Relief et } \\
\text { pentes }\end{array}$ & Sols & Utilisation & $\begin{array}{l}\text { Caractéris- } \\
\text { tiques addi- } \\
\text { tionnelles }\end{array}$ & $\begin{array}{l}\text { Sym- } \\
\text { bole }\end{array}$ \\
\hline $\begin{array}{l}\text { d'origine } \\
\text { morainique }\end{array}$ & terrasses & de viticulture $(\mathrm{Fa})$ & viticulture & $\begin{array}{l}\text { très infl. } p . \\
\text { viticulture }\end{array}$ & Fac \\
\hline \multirow{3}{*}{$\begin{array}{l}\text { roches et dé- } \\
\text { pôts de pente }\end{array}$} & convexe (c) & Regosols, pierreux à $25 \mathrm{~cm}$ & forêts & - & $\mathrm{Fcl}, \mathrm{Kcl}$ \\
\hline & \multirow[t]{2}{*}{ concave (d) } & id. pierreux à $50 \mathrm{~cm}$ & prés & & $\mathrm{Kd} 2$ \\
\hline & & $\begin{array}{l}\text { Cambisols, argile pierreuse } \\
\text { à } 50 \mathrm{~cm}\end{array}$ & idem & & Fd4 \\
\hline \multirow[t]{3}{*}{$\begin{array}{l}\text { d'origine } \\
\text { morainique }\end{array}$} & convexe (h) & $\begin{array}{l}\text { Cambisols limoneux, } \\
\text { pierreux à } 50 \mathrm{~cm}\end{array}$ & prés & & $\mathrm{Kh} 4, \mathrm{Ph} 4$ \\
\hline & concave (j) & Regosols, pierreux à $50 \mathrm{~cm}$ & forêts & - & Fj1 \\
\hline & irrégulier (k) & $\begin{array}{l}\text { Cambisols limoneux, } \\
\text { pierreux à } 50 \mathrm{~cm}\end{array}$ & prés & - & Kk4 \\
\hline $\begin{array}{l}\text { dépôts fluvio- } \\
\text { glaciaires }\end{array}$ & concave (1) & $\begin{array}{l}\text { Cambisols + Luvisols argi- } \\
\text { leux, pierreux et calcaires } \\
\text { à } 50 \mathrm{~cm}\end{array}$ & forêts & & $\mathrm{F} 17$ \\
\hline
\end{tabular}




\begin{tabular}{|c|c|c|c|c|c|}
\hline \multicolumn{6}{|c|}{ Pentes: modérées ( $5 \%$ à $10 \%)(\mathrm{G}, \mathrm{L}, \mathrm{R})$} \\
\hline $\begin{array}{l}\text { d'origine } \\
\text { morainique }\end{array}$ & terrasses & de viticulture (GA) & viticulture & $\begin{array}{l}\text { très infl. } p . \\
\text { viticulture }\end{array}$ & Gac \\
\hline $\begin{array}{l}\text { roches et } \\
\text { matériaux } \\
\text { de pente }\end{array}$ & rectiligne (b) & $\begin{array}{l}\text { Cambisols, argile, calcaire, } \\
\text { pierreux à } 50 \mathrm{~cm}\end{array}$ & prés & - & Gb4 \\
\hline $\begin{array}{l}\text { roches, maté- } \\
\text { riaux de pente } \\
\text { et morainiques }\end{array}$ & irrégulier (f) & $\begin{array}{l}\text { Lithosols + Regosols limo- } \\
\text { neux, pierreux à } 25 \mathrm{~cm} \\
+ \text { Cambisols limoneux, } \\
\text { pierreux à } 50 \mathrm{~cm}\end{array}$ & prés & karstique & $\begin{array}{l}\text { Lf7, 6, } \\
\text { Rf7, } 6\end{array}$ \\
\hline \multirow[t]{2}{*}{$\begin{array}{l}\text { d'origine } \\
\text { morainique }\end{array}$} & \multirow[t]{2}{*}{ irrégulier (k) } & Regosols pierreux à $50 \mathrm{~cm}$ & $\begin{array}{l}\text { cultures } \\
\text { habitations }\end{array}$ & & Gk1 \\
\hline & & $\begin{array}{l}\text { Cambisols limoneux, } \\
\text { pierreux à } 50 \mathrm{~cm}\end{array}$ & prés & & Lk4 \\
\hline \multicolumn{6}{|c|}{ Pentes: douces (moins de $5 \%)(\mathrm{H})$} \\
\hline \multirow[t]{2}{*}{$\begin{array}{l}\text { dépôts fluvio- } \\
\text { glaciaires }\end{array}$} & terrasses $(\mathrm{ad})$ & de viticulture & viticulture & $\begin{array}{l}\text { très infl. } p . \\
\text { viticulture }\end{array}$ & Had \\
\hline & $\begin{array}{l}\text { pentes } \\
\text { douces }\end{array}$ & Regosols pierreux à $25 \mathrm{~cm}$ & $\begin{array}{l}\text { forêts et } \\
\text { prés }\end{array}$ & & $\mathrm{He} 2$ \\
\hline
\end{tabular}

A partir d'un extrait de la carte écopaysagique, trois cartes de vocation pour des objectifs différents ont été dessinées: viticulture, campings et résidences secondaires. Il ne s'agit là que de premiers croquis, non encore contrôlés par des experts, mais qui indiquent différents «types d'utilisation des terres» (vINK, 1975). La méthodologie de l'évaluation des terres est encore en train de se développer, comme l'est également notre connaissance de quelques régions de Suisse romande. Aussi espérons-nous continuer nos études sur ces questions. Nos recherches sont facilitées par la beauté des paysages helvétiques et par la coopération de nos collègues suisses, trop nombreux pour être tous cités, à qui nous exprimons notre profonde gratitude.

\section{Bibliographie}

BLANKERS, J., 1975, Landschapsecologische Kaart van het Gebied van Montricher, Thèse de 2ème cycle, Lab. Géogr. Phys. et Pédol., Univ. Amsterdam.

BRINKMAN, R. and SMYTH, A. J. (Ed), 1973, Land evaluation for rural purpose, Publ. 17, Intern. Inst. Land Reclam. and Improvement (ILRI), Wageningen.

BURRI, M., 1962, Les dépôts quaternaires de la vallée du Rhône entre St-Maurice et le Léman, Bull. Lab. Géol. Univ. Laus. 132, Lausanne.

CHORLEY, R. J. and KENNEDY, B. A., 1971, Physical geography, a systems approach. Prentice Hall, London. DE HAAS, W. G. L. et RIJNBERG, Th. F. (Ed), 1973, Proceedings of the International Symposium on Integrated Surveys and Environmental management in developing countries, ITC Journal, 1973-2, Enschede.

DUDAL, R., 1970, Key to the Units of the soil map of the world, FAO, Rome. 
DU PRÉE, E.A., 1975, Landschapsecologische Kartering langs de vostvever van het meer van Neuchâtel, Thèse $2{ }^{\mathrm{ème}}$ cycle Lab. Géogr. Phys. et Pédol,Univ. Amsterdam. FREI, E., JUHASZ, P., BACH, R., 1966, Bodenkarte der Schweiz 1:1000000, Schweiz. Landw. Forsch. V, p. $537-551$.

FREI, E., et al., 1969, Bodenkarten unterstützen Meliorationen, Bonitierungen und Planungsarbeiten, Mitt. Schweiz. Landw. 17, p. 197-208.

HAEBERLI, R., 1968, Levé cartographique agricole des stations végétales de la Côte (Canton de Vaud, Suisse), Thèse 4196, ETH, Zürich.

HAEBERLI, R., 1971, a) Carte des dangers de gel tardif printanier dans le Canton de Vaud, Cah. amén. rég. 11, Off. Vaud. Urban., Lausanne.

b) Carte écologique-physiographique des sols du Canton de Vaud, C.a.r. 12, Off. Vaud. Urban., Lausanne.

c) Carte du potentiel naturel des surfaces agricoles du Canton de Vaud, C.a.r. 13, Off. Vaud. Urban., Lausanne. HANTKE, R., 1963, Chronologische Probleme im schweizerischen Quartär, Jbr. u. Mitt. oberrl. geol. Ver. NF 45, p. $45-60$.

JUNGERIUS, P. D., KOSTER, E. A. et KWAAD, F. J.P. M. (ED.), 1973, Fysische Geografie, Oosthoek, Utrecht.

KLAASEN, I., 1974, Het gebied rond Aigle (Vaud, Zwitserland), Thèse de 2ème Cycle, Lab. Géogr. Phys. et Pédol., Univ. Amsterdam.

MARR, R. L., 1970, Geländeklimatische Untersuchung im Raum südlich von Basel, Basl. Beitr. Geogr., Heft 12, Basel.

MEYER DE STADELHOFEN, M.C., 1967, Exemples de contributions de la géophysique aux travaux de l'ingénieur, Bull. Techn. Suisse Romand, № 10 (1967), p. 1-5.

PORTMANN, J.P., 1955, Pétrographie des moraines du glacier Würmien du Rhône dans la région des lacs subjurassiens (Suisse), Bull. Soc. Neuch. Géogr. 51, p. 14-55.

PORTMANN, J.P., 1966, Pétrographie des formations glaciaires à l'est du lac de Brévine (Suisse), Eclogae Geol. Helv. 59, p. 697-721.
PORTMANN, J.P., 1972, Pour une géographie globale, Bull. Soc. Neuch. Géogr. 54, p. 3-17.

SCHREIBER, K. F., 1968, Les conditions thermiques du Canton de Vaud, Off. Cant. Urb., Lausanne.

SCHWAB, H., 1964, Archäologische Entdeckungen im Rahmen der 2. Juragewässer-Korrektion, Wasser- und Energiewirtschaft 11, p. 3-7.

SCHWAB, H. und MÜLLER, R., 1973, Die Vergangenheit des Seelandes in neuem Licht, Universitätsverlag, Freiburg, Schweiz.

SOIL SURVEY STAFF, 1973, Soil Taxonomy, Preliminary Abridged Text, U.S. Dept. Agric. Washington D. C.

TROLL, C., 1966, Landscape Ecology, Publ. S4, ITC-UNESCO Centre for Integrated Surveys, Delft.

VAN DER MEER, J. J. M., 1973, Een Bodemkartering in de omgeving van Murten, Zwitserland, Thèse 2ème cycle, Lab. Géogr. Phys et Pédol., Univ. Amsterdam.

VAN DER MEER, J. J. M., 1975, Bull. Soc. Sci. Nat., Fribourg (en préparation).

VINK, A.P. A., 1963, Aspects de Pédologie Appliquée, A la Baconnière, Neuchâtel.

VINK, A. P. A., 1968, Aerial photographs and the Soil Sciences, in: P. REY (Ed.) Aerial photographs and integrated studies, UNESCO, Paris.

VINK, A. P. A. and SEVINK, J., 1974, Soils and Paleosols in the Lutterzand, Meded. Rijks Geol. Dienst, Haarlem, N. S. p. $165-185$.

VINK, A. P. A., 1973, Ricerche sui suoli e i paesaggi nel Lazio e in Toscana, Rivista Geografica Italiana 80, p. 261-277.

VINK, A.P. A., 1974, La géographie physique: Science naturelle et appliquée, Bull. Soc. Neuch. Géogr. LIX, 3, p. 11-24.

VINK, A. P. A., 1975, Land use in advancing agriculture, Springer Verlag, Berlin/Heidelberg/New York.

WEVERS, R., 1974, Verslag van een landschaps-ecologische Kartering in het gebied van Orbe, Kanton Vaud, Zwitserland, Thèse 2ème cycle, Lab. Géogr. Phys. et Pédol., Univ. Amsterdam. 